\title{
Snail heterogeneity in clear cell renal cell carcinoma
}

Laura Zaldumbide ${ }^{1}$, Asier Erramuzpe ${ }^{2}$, Rosa Guarch ${ }^{3}$, Rafael Pulido ${ }^{4,5}$, Jesús M. Cortés $2,5,6$ and José I. López ${ }^{1,4^{*}}$

\begin{abstract}
Background: Intratumor heterogeneity may be responsible of the unpredictable aggressive clinical behavior that some clear cell renal cell carcinomas display. This clinical uncertainty may be caused by insufficient sampling, leaving out of histological analysis foci of high grade tumor areas. Although molecular approaches are providing important information on renal intratumor heterogeneity, a focus on this topic from the practicing pathologist' perspective is still pending.

Methods: Four distant tumor areas of 40 organ-confined clear cell renal cell carcinomas were selected for histopathological and immunohistochemical evaluation. Tumor size, cell type (clear/granular), Fuhrman's grade, Staging, as well as immunostaining with Snail, ZEB1, Twist, Vimentin, E-cadherin, $\beta$-catenin, PTEN, p-Akt, p110a, and SETD2, were analyzed for intratumor heterogeneity using a classification and regression tree algorithm.

Results: Cell type and Fuhrman's grade were heterogeneous in 12.5 and $60 \%$ of the tumors, respectively. If cell type was homogeneous (clear cell) then the tumors were low-grade in $88.57 \%$ of cases. Immunostaining heterogeneity was significant in the series and oscillated between $15 \%$ for p110a and $80 \%$ for Snail. When Snail immunostaining was homogeneous the tumor was histologically homogeneous in $100 \%$ of cases. If Snail was heterogeneous, the tumor was heterogeneous in $75 \%$ of the cases. Average tumor diameter was $4.3 \mathrm{~cm}$. Tumors larger than $3.7 \mathrm{~cm}$ were heterogeneous for Vimentin immunostaining in $72.5 \%$ of cases. Tumors displaying negative immunostaining for both ZEB1 and Twist were low grade in $100 \%$ of the cases.
\end{abstract}

Conclusions: Intratumor heterogeneity is a common event in clear cell renal cell carcinoma, which can be monitored by immunohistochemistry in routine practice. Snail seems to be particularly useful in the identification of intratumor heterogeneity. The suitability of current sampling protocols in renal cancer is discussed.

Keywords: Clear cell renal cell carcinoma, Intratumor heterogeneity, Epithelial to mesenchymal transition, Immunohistochemistry, Snail, Tumor sampling

\section{Background}

Renal cancer is among the most common malignancies in men and women in Western countries, with more than 61000 estimated new cases in the United States in 2015 [1]. Clear cell renal cell carcinoma (CCRCC) is the most frequent renal cancer histologic subtype, accounting for about $70 \%$ of renal carcinomas [2]. CCRCC management is problematic for urologists and

\footnotetext{
* Correspondence: joseignacio.lopez@osakidetza.eus

'Department of Pathology, Cruces University Hospital, University of the Basque Country (UPV/EHU), Plaza de Cruces s/n, 48903 Barakaldo, Bizkaia, Spain

${ }^{4}$ Biomarkers in Cancer Unit, Biocruces Health Research Institute, Barakaldo, Bizkaia, Spain

Full list of author information is available at the end of the article
}

oncologists. This neoplasm follows a quite unpredictable clinical course and usually displays resistance to radio- and chemotherapy, surgery being the only effective treatment to date.

CCRCC is a paradigm of an intrinsically heterogeneous neoplasm and most problems with its clinical management rely on this point. Intratumor heterogeneity (ITH) in CCRCC follows a spatial and temporal branched pattern [3-5], with multiple cell clones evolving independently from each other during tumor evolution. This fact reflects the complexity of tumor biology [6] and adds further difficulties to define effective targeted treatments against this neoplasm [7]. CCRCC ITH has been specifically studied in the last years from a 
molecular perspective [3, 4, 7-9]. However, there are few studies addressing the problem from a clinical routine approach [10-12].

The aim of this study was to evaluate the ITH of the expression of several epithelial to mesenchymal transition (EMT) markers and PI3K/PTEN/Akt-pathway markers, as assessed by immunohistochemistry, and its relationship with standard histopathological prognosis parameters in a series of 40 CCRCC collected prospectively.

\section{Methods}

The authors declare that all the experiments carried out in this study comply with current Spanish and European Union legal regulations, and have been approved by the Ethical and Scientific Committees of the Basque Country Public Health System (Osakidetza) (CEIC-E 2015/060).

Forty organ-confined CCRCCs from two Spanish medical institutions were included prospectively in the study from September 2011 to June 2012. Cases showing gross hemorrhage, necrosis or hard whitish areas indicative of sarcomatoid transformation were excluded. All the cases were collected prospectively, diagnosed and classified by the same pathologist in each hospital following similar criteria. Clinical data were retrieved from medical histories.

On gross examination, four distant zones were sampled in each tumor and then formalin-fixed and paraffinembedded following routine methods. Whenever possible, samples were obtained in the cardinal points of the largest tumor slice and had to be similar to the naked eye. Once the resulting hematoxylin-eosin (HE) slides were histologically reviewed, a $2.5 \mathrm{~mm}$-in-diameter tumor sample was selected in each block to build tissue microarrays (TMA). TMA were performed with the resulting 160 tumor samples (4 areas each case, 40 cases). Samples were placed randomly in the TMA to assure blind evaluation. Each TMA contained an internal control.

Standard histopathologic data were evaluated on HE stained slides (Fig. 1), including cell-type (clear vs. granular), Fuhrman's grade [classical (G1, G2, G3 and G4) and grouped in low (G1/2) and high (G3/4) grades], tumor diameter, and pT UICC staging (pT1a, pT1b and pT2). Tumors were considered histologically heterogeneous when at least one of the four samples of each tumor in the TMA had different cell type and/or grade.

Immunohistochemical staining was performed in automated immunostainers (EnVision FLEX, Dako Autostainer Plus, Dako, Glostrup, Denmark; and BenchMark Ultra, Ventana Medical Systems, Tucson, AZ, USA) following routine methods. Tris-EDTA was used for antigen retrieval in all cases. Negative controls were slides not exposed to the primary antibody, and these were incubated in PBS and then processed under the same conditions as the test slides. The analysis was performed using a Nikon Eclipse 80i microscope (Tokyo, Japan). A panel of antibodies against EMT markers (Snail, Twist, ZEB1, $\beta$-catenin, E-cadherin, and Vimentin), PI3K/ PTEN/Akt pathway-related markers (PTEN, p110 $\alpha$, pAkt), as well as SETD2, was tested (Fig. 2). Table 1 shows the source and working dilutions of these antibodies. Heterogeneous immunostaining category was assigned

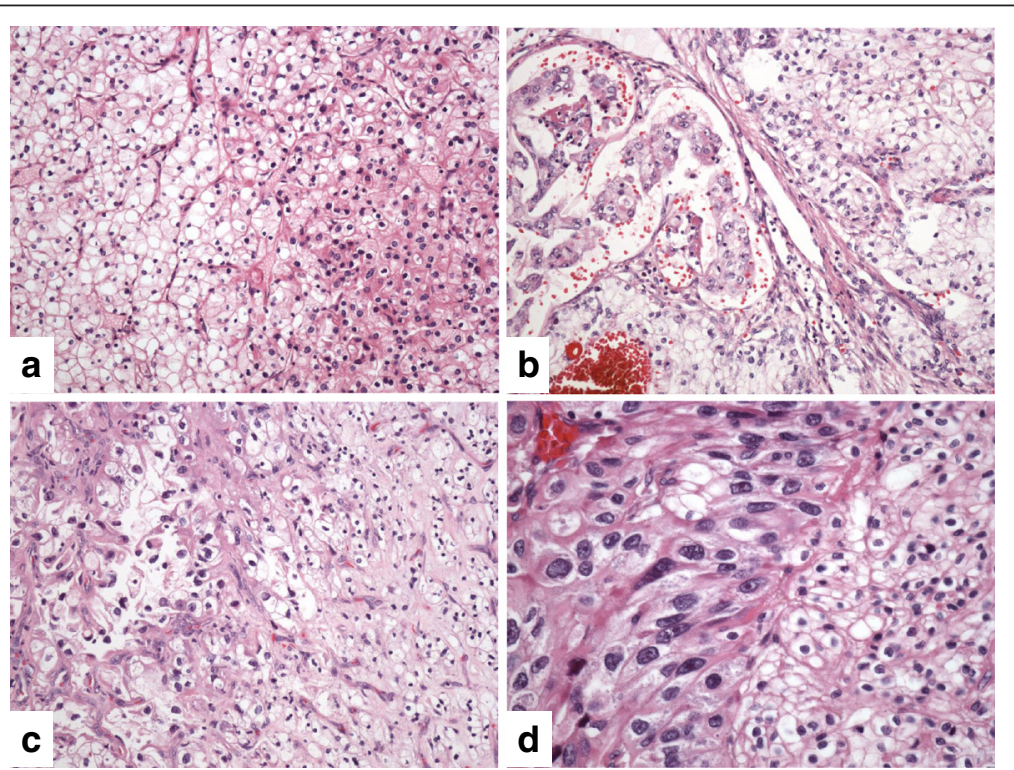

Fig. 1 Intratumor heterogeneity in adjacent tumor areas of clear cell renal cell carcinomas on routine hematoxylin-eosin slides. a Clear and granular cells, b Papillary and solid cell tumor architecture, c High (papillary) and low (solid) Fuhrman's grades, d High and low Fuhrman's grades in clear cells (solid pattern) 

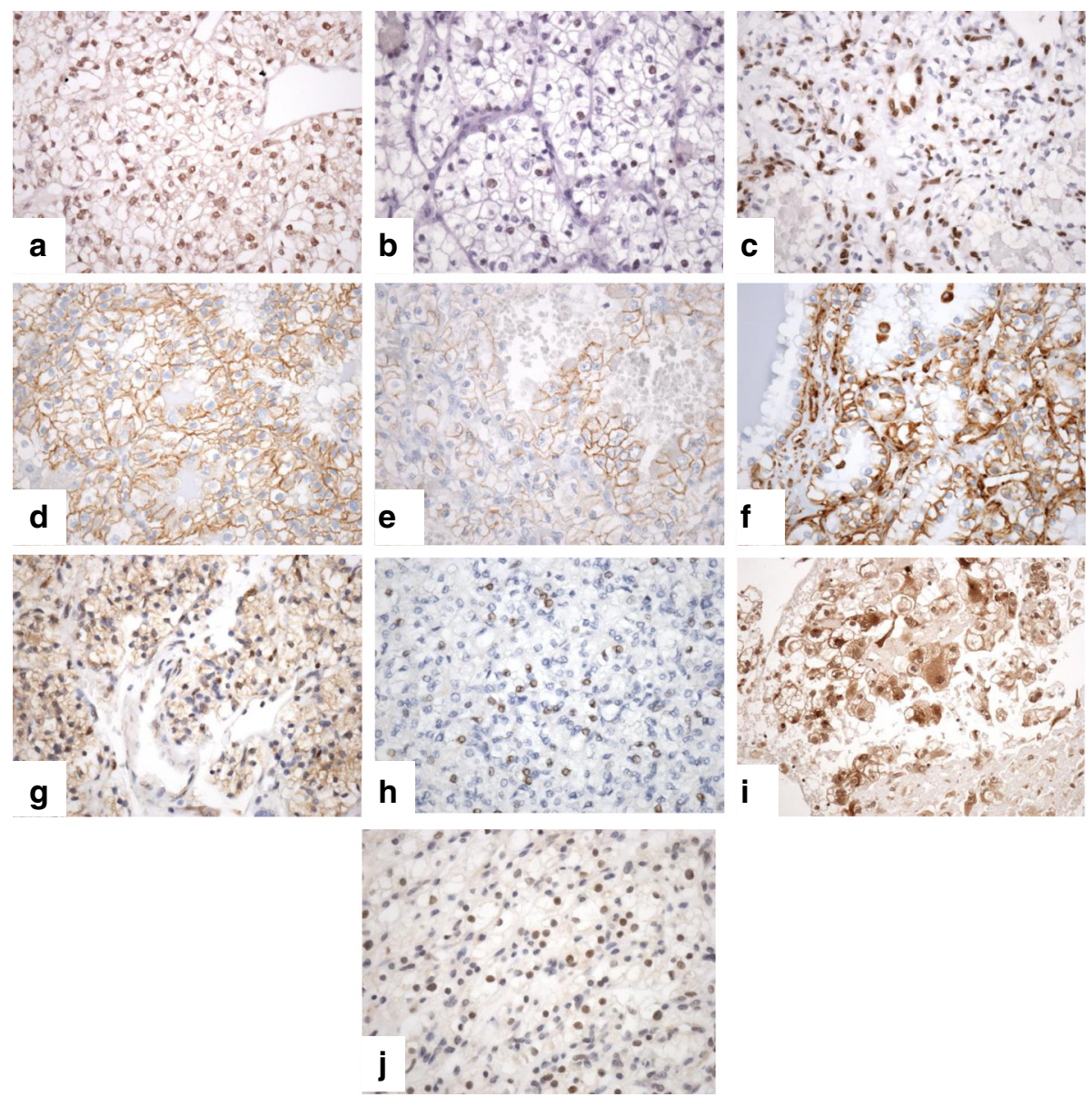

Fig. 2 Immunohistochemical pattern in clear cell renal cell carcinomas displaying nuclear and/or cytoplasmic and/or membranous staining. a Snail, b Twist, c ZEB1, d $\beta$-catenin, e E-cadherin, f Vimentin, $\mathbf{g}$ PTEN, h p110a, i p-Akt, j SETD2

when positive and negative immunostainings were detected among the four samples of each tumor. Cases were considered homogeneous when the four samples of each tumor displayed the same immunohistochemical profile, and could be positive or negative.

Table 1 List of antibodies tested in the study

\begin{tabular}{lllll}
\hline Ab & Source/code & & & Dilution \\
\hline Snail & Abcam, ab180714 & polyclonal & $\uparrow \mathrm{pH}$ & 1:75 \\
Twist & Santa Cruz, sc15393 & polyclonal & $\downarrow \mathrm{pH}$ & 1:250 \\
ZEB1 & Santa Cruz, sc25388 & polyclonal & $\uparrow \mathrm{pH}$ & 1:100 \\
B-catenin & Cell Marque, 760-4242 & monoclonal & $\uparrow \mathrm{pH}$ & prediluted \\
E-cadherin & Roche, Ventana, 790-4497 & monoclonal & $\uparrow \mathrm{pH}$ & prediluted \\
Vimentin & Roche, Ventana, 790-2917 & monoclonal & $\uparrow \mathrm{pH}$ & prediluted \\
PTEN (6H2) & Dako, M3627 & monoclonal & $\uparrow \mathrm{pH}$ & $1: 50$ \\
P110a & Pierce, PA5-27192 & polyclonal & $\downarrow \mathrm{pH}$ & $1: 500$ \\
Akt & Life Technologies, 700392 & monoclonal & $\uparrow \mathrm{pH}$ & $1: 500$ \\
SETD2 & Pierce, PA5-34934 & polyclonal & $\uparrow \mathrm{pH}$ & 1:500 \\
\hline
\end{tabular}

In a preliminary analysis, an independent 2-group $t$ test was performed to check if age, sex or tumor size were different in the two conditions, heterogeneous vs. homogeneous ( $t$ test function in $\mathrm{R}, \mathrm{r}$-project.org). Afterwards, we performed classification and attribute selection methods for ITH. First, we chose ITH as the response variable to perform classification, coding it in a binary variable with two values, to be heterogeneous or not. Next, a classification and regression tree (CART) algorithm was performed using a 10-fold stratified crossvalidation for testing. In particular, the CART algorithm was applied using the Waikato Environment for Knowledge Analysis (WEKA) [13]. Clinical, histological and immunohistochemical descriptors were selected to obtain the classification rules for the heterogeneity response; in particular, we used age, sex, simple Fuhrman's grade (grades from 1 to 4), grouped Fuhrman's grade [low grade (G1/2) and high grade (G3/4)], tumor diameter, pT staging, cell type (clear vs. granular) and immunostaining results of all the antibodies tested. 
The use of WEKA also allowed us to discriminate the relevant descriptors (those really affecting the classification performance) from the irrelevant ones (those which did not contribute to the classification performance). Attribute selection was performed using three different algorithms (a best-first search, a rank-search, and a random-search) and the results did not differ between the three methods.

\section{Results}

There was a male predominance in the series $(30 \mathrm{M} /$ $10 \mathrm{~F}$ ) with an average age of 62.3 years (range, 33-88). Tumor diameter oscillated between 1 and $10 \mathrm{~cm}$ (average, $4.3 \mathrm{~cm}$ ). Following the aforementioned methodology, all the cases were organ confined and pT distribution was as follows: pT1a, 21 cases (52.5\%); pT1b, 17 cases (42.5\%); pT2: 2 cases (5\%). On HE stained sections 35 cases $(87.5 \%)$ were homogeneous and 5 heterogeneous $(12.5 \%)$ with respect to cell type. All the homogeneous cases were composed of conventional clear cells. Heterogeneous cases combined clear and granular cells. No case composed exclusively by granular cells was identified. Fuhrman's grade [14] was homogeneous in the four samples in 16 cases (40\%), 11 of them being G1 and $5 \mathrm{G} 2$, and heterogeneous in 24 cases $(60 \%)$. Grouped grading distribution displayed 32 homogeneous $(80 \%)$ and 8 heterogeneous $(20 \%)$ cases. All homogeneous cases in the series were low grade (G1/2) tumors (100\%).

Immunohistochemical results for ITH are summarized in Table 2. Briefly, Snail immunostaining was heterogeneous in $80 \%$ of cases (32/40), Twist in $40 \%(16 / 40)$, ZEB-1 in $62.5 \%(25 / 40), \beta$-catenin in $37.5 \%(15 / 40)$, Ecadherin in $37.5 \%(15 / 40)$, Vimentin in $50 \%(20 / 40)$, PTEN in $35 \%$ (14/40), p110 $\alpha$ in $15 \%(6 / 40)$, p-Akt in $30 \%(12 / 40)$, and SETD2 in $42.5 \%$ (17/40). Immunostaining pattern did not show any difference between G3 and G4 areas. Snail immunostaining showed a relation

Table 2 Immunohistochemical results

\begin{tabular}{llll}
\hline & \multicolumn{2}{l}{ Homogeneous } & Heterogeneous \\
\cline { 2 - 3 } Abs & Positive & Negative & \\
\hline Snail & 8 & 0 & $32(80 \%)$ \\
Twist & 1 & 23 & $16(40 \%)$ \\
ZEB-1 & 1 & 14 & $25(62.5 \%)$ \\
B-catenin & 22 & 3 & $15(37.5 \%)$ \\
E-cadherin & 0 & 25 & $15(37.5 \%)$ \\
Vimentin & 18 & 2 & $20(50 \%)$ \\
PTEN (6H2) & 0 & 26 & $14(35 \%)$ \\
P110a & 34 & 0 & $6(15 \%)$ \\
Akt & 0 & 28 & $12(30 \%)$ \\
SETD2 & 23 & 0 & $17(42.5 \%)$ \\
\hline
\end{tabular}

with Fuhrman's grade: all high grade (G3/4) CCRCC were Snail heterogeneous and all Snail homogeneous CCRCC were low grade tumors (G1/2). Also, ZEB1 and Twist immunostainings were correlated with grade: if both ZEB1 and Twist immunostaining was negative, then tumor grade was low in $100 \%$ of the cases.

We obtained three different classification results from applying the CART algorithm to different response variables (Table 3). Although in general the output of a CART algorithm might be a decision tree with multiple branches, for the particular situation of our data sets, trees only had 2 branches, resulting in only one determinant variable for the three particular situations. First, considering all possible descriptors and ITH as the response variable, the decision tree of the CART classification showed that the only classifier variable was Snail immunostaining. Thus, if Snail immunostaining was homogeneous, the tumor was histologically homogeneous in all cases. By contrast, if Snail immunostaining was heterogeneous, the tumor was histologically heterogeneous in $75 \%$ of cases. Using this classification scheme, $80 \%$ of the cases in the series were correctly classified. Any other variable, alone or in combination, was not relevant for ITH.

A second CART classification was performed considering Grade as the response variable. In this case, cell type (clear vs. granular) variable was the best one to classify the response. If cell type was 'clear' (group 1 in Table 3), then Grade was classified as G1-G2 with $88.57 \%$ of correct classified instances. If cell type was a mixture of 'clear and granular' (group 2 in Table 3), then the Grade was G3-G4 with $80 \%$ of correctness. Using this classification scheme, $87.5 \%$ of the cases in the series were correctly classified. Any other variable, alone or in combination, was not relevant for heterogeneity in Grade classification.

Finally, a third CART classification analysis was performed choosing tumor diameter, Grade, grouped Grade and cell type, and combinations of them, as descriptors, and the rest of variables as response variables. This analysis showed that Vimentin was the only variable classified with correctness higher than $66 \%$. The rest of variables gave a poorer performance. This analysis unveiled that those samples with a tumor diameter larger than $3.7 \mathrm{~cm}$ were heterogeneous for Vimentin immunostaining with an accuracy of $72.5 \%$.

\section{Discussion}

ITH is an inherent phenomenon in carcinogenesis that is being intensively analyzed using massive sequencing tools in many human neoplasms, kidney included [3-5, 7-9, 15]. However, pathological studies related with ITH under daily routine conditions are scarce. At this respect, Nassar et al. [16] documented 
Table 3 CART classification results over three different response variables (first column) revealed three different determinant variables (second column)

\begin{tabular}{|c|c|c|c|c|}
\hline \multirow[t]{2}{*}{ Response variable } & \multirow[t]{2}{*}{ Determinant variable } & Individual Correctness & Individual Correctness & \multirow{2}{*}{$\begin{array}{l}\text { Total } \\
\text { Correctnes }\end{array}$} \\
\hline & & 1 & 2 & \\
\hline \multirow[t]{2}{*}{ Grade Heterogeneity } & \multirow[t]{2}{*}{ Snail immunostaining } & Snail het -> Grade het & Snail hom - > Grade hom & $80 \%$ \\
\hline & & $75 \%(24 / 32)$ & $100 \%(8 / 8)$ & $(32 / 40)$ \\
\hline \multirow[t]{2}{*}{ Grade } & \multirow[t]{2}{*}{ Cell type } & $\begin{array}{l}\text { Clear cell (hom) - > Grade } \\
\text { low (G1/G2) }\end{array}$ & $\begin{array}{l}\text { Granular cell (het) - > Grade } \\
\text { high (G3/G4) }\end{array}$ & $87.5 \%$ \\
\hline & & $88.57 \%(31 / 35)$ & $80 \%(4 / 5)$ & $(35 / 40)$ \\
\hline \multirow[t]{2}{*}{ Diameter } & \multirow[t]{2}{*}{ Vimentin } & Diam $>3.7$ - > Vim het & Diam $<=3.7->$ Vim hom & $72.5 \%$ \\
\hline & & $72.72 \%(16 / 22)$ & $72.22 \%(13 / 18)$ & $(29 / 40)$ \\
\hline
\end{tabular}

Individual Correctness (columns 3 and 4) shows the classification performance with respect to each group of the response variable and Total Correctness (column 5) shows the overall classification performance applied to the entire dataset

ITH in breast cancer by immunohistochemistry and highlighted the negative clinical impact of potential false negative immunohistochemical results obtained after a study performed in small, probably non representative, core biopsies. In renal cancer this is an important issue since renal tumors are frequently big in size, and current protocols of tumor sampling recommend the selection for microscopic study of one block per centimeter of tumor plus an additional sample of every suspicious area on naked eye [17-19]. Following these guidelines, more than $90 \%$ of the neoplastic tissue in many renal tumors may escape the pathologist's routine analysis. This fact makes difficult to quantify the nature and extent of ITH, if present, in most CCRCC. Although pathologists recognize by naked eye whitish hard areas related to sarcomatoid transformation, as well as hemorrhagic and/or necrotic foci, there is a significant amount of ITH hidden in apparently homogeneous areas, as recently reported [10-12]. Furthermore, an incomplete tumor sampling may justify the unexpected aggressive behavior that some lowgrade CCRCC show [12]. The present work has shown the relationship between cell type and Grade in CCRCC, since a mixture of conventional clear and granular cells determines high tumor Grade (G3/G4) in more than $83 \%$ of cases.

Two recent studies on CCRCC have contributed to know more about ITH from a practical perspective. The first one intended to identify how much information is lost in routine practice after following the official sampling protocols. For such a purpose, a total tumor sampling, an unsuitable strategy in daily routine, was performed in 47 prospective CCRCC, and the histological analysis revealed a significant higher number of high grade tumors than with conventional sampling [10]. This finding raises serious doubts about the appropriateness of currently accepted CCRCC sampling protocols, suggesting that in some histopathological examinations data potentially crucial for the patient might be ignored. The second study used data mining tools and showed that only CCRCC tumors with less than $3.8 \mathrm{~cm}$ in diameter are always histologically homogeneous [12]. In addition, the present report shows that CCRCC tumors with more than $3.7 \mathrm{~cm}$ display ITH for Vimentin immunostaining. Interestingly, both size numbers are very close to the pathological staging frontier between $\mathrm{pT} 1 \mathrm{a}$ and $\mathrm{pT} 1 \mathrm{~b}$, suggesting that $4 \mathrm{~cm}$ in diameter is the size in which ITH appears visible on both histological and immunohistochemical settings. This finding, however, does not infer that ITH at gene mutation status level follows the same tendency.

The present work was also designed to investigate the utility of immunohistochemistry in the identification of ITH in CCRCC. The panel of protein markers used was selected considering their potential roles in the development of tumor aggressiveness, tumor diversification and metastatic processes.

EMT is a cellular mechanism extensively reviewed in the literature $[3,9,20,21]$. The process explains how, under certain conditions, epithelial cells transform into mesenchymal cells. Briefly, EMT includes first the loss of the apical-basal polarity and cell-to-cell lateral junctions and then the acquisition of spindle cell shape, endto-end polarity, and migration abilities. This change occurs in three different settings: embryonic development, tissue repair and neoplasia. The EMT concept is indispensable to understand the invasive properties and the metastatic capacities of carcinomas [22-27]. Interestingly, EMT may be a reversible process, what is called mesenchymal-to-epithelial transition (MET), which partially explains why high grade carcinomas may appear in distant metastases with an extremely low grade phenotype $[22,23,28]$.

The immunohistochemical expression of several EMT markers in renal cell carcinomas has been previously reported. For instance, Clusterin and Twist expression has been related with tumor aggressiveness [29], tumor 
recurrence [30], and poor prognosis [29, 31]. Our study has focused on the immunohistochemical distribution of seven EMT markers [including cell-surface (E-cadherin) markers, cytoskeletal and cytoskeletal-associated (vimentin, $\beta$-catenin) proteins, and transcription factors (Snail, ZEB1, Twist, $\beta$-catenin)] in the context of ITH in CCRCC.

The Snail antibody tested in this study recognizes both Snail1 and Snail2. Snail1 is a zinc-finger transcription factor directly involved in the repression of E-cadherin transcription thus promoting the acquisition of a mesenchymal phenotype [28, 32]. Snail1 seems to be activated by ghrelin, an appetite-regulating molecule that promotes growth hormone release [33]. The immunohistochemical expression of Snail1 in CCRCC has been associated to advanced stage, high grade, local invasion and metastases [34]. A recent study has confirmed that Snail1 immunostaining predicts early recurrence and poor survival in CCRCC patients [35]. In our series, all high grade CCRCC tumors, including 3 G4 cases, displayed positive immunostaining for Snail. By contrast, Snail negative CCRCC were always low-grade. In addition, we have found that Snail positive immunostaining determines ITH, as defined by pathological analysis. The effect of Snail1 in promoting EMT can be suppressed by Klotho, as demonstrated by Zhu et al. in a recent study [36]. Klotho, an anti-aging and tumor suppressor protein expressed in renal tubular cells, inhibits PI3K/Akt/GSK3 $\beta /$ Snail signaling thus suppressing EMT, tumor invasion and migration. How the Snail1-associated transcriptional cell profile may be related with ITH in CCRCC remains to be investigated.

Twist is a transcription factor classically involved in embryonic development [37]. In addition, Twist plays a role in tumor growth, cell invasion and metastases by regulating neoangiogenesis and extracellular matrix degradation [29]. Twist expression is associated with bad prognosis in CCRCC $[29,30]$. We have found heterogeneous Twist immunostaining in 16 CCRCC (40 \%) and homogeneously positive staining in 1 case, although Twist was an irrelevant variable for classification of ITH and grade.

ZEB1 and ZEB2 are zinc-finger E-box binding transcription factors crucial for the activation of EMT [21]. $Z E B 1$ gene is up-regulated in primary renal tumors compared with their metastases, suggesting a reversal MET process in the metastatic seed [38]. The miRNA-200 family is directly involved in EMT and MET processes targeting the E-cadherin repressors ZEB1 and ZEB2 $[28,39]$. Interestingly, CCRCC show down-regulation of all the members of this miRNA family [40], a fact that may be useful in the differential diagnosis of CCRCC [41, 42]. In our study, ZEB1 immunostaining was heterogeneous in 25 cases $(62.5 \%)$ and homogeneously positive in 1 case, but after attribute selection methods, ZEB1 immunostaining was a non-relevant variable for ITH classification as well as for tumor grade classification. However, a combined negative immunostaining for ZEB1 and Twist was always associated to low grade (G1/2) tumors, suggesting that the combined analysis of these two markers could be informative for CCRCC prognosis.

$\beta$-catenin is a cytoplasmic protein that links cadherins to cytoskeleton. Additionally, it serves as a cotranscriptional activator in the nucleus [43]. $\beta$-catenin is expressed in neoplastic cells undergoing EMT, especially at the stromal invasion front, as reported in colorectal adenocarcinoma [44]. $\beta$-catenin dysregulation has been associated to aggressive clinical course and shorter survival in CCRCC [45]. A heterogeneous immunohistochemical expression of this protein has been demonstrated in 15 cases in the present series, but this number has not reached any significance in the CART algorithms. In addition, $\beta$-catenin immunostaining was diffusely positive (nucleus and cytoplasmic membrane) in 22 cases and totally negative in 3 cases.

E-cadherin is expressed at the surface of epithelial cells in normal conditions, decreasing in abundance during EMT process in a switch with $\mathrm{N}$-cadherin [46]. Ecadherin immunostaining is completely lost in $60 \%$ of our cases. By contrast, focal and diffuse immunostaining was detected in 15 and 1 cases, respectively. After attribute selection methods, E-cadherin immunostaining was not a relevant variable for ITH.

Vimentin is an intermediate filament that has been implicated in all EMT scenarios (embryonal development, tissue repair and cancer progression) [28]. Low mRNA levels of Vimentin correlate with a better outcome of CCRCC patients [38]. From a practical viewpoint, Vimentin is usually co-expressed with cytokeratins and CD10, helping in the routine differential diagnosis of renal tumors [47]. The pathologist's experience reveals that Vimentin immunostaining is sometimes patchy in CCRCC. In our study, Vimentin immunostaining was heterogeneous in half of the cases $(20$ cases), being homogeneously positive in 18 cases and totally negative in 2 cases. As previously mentioned, our classification analysis reveals a relation between tumor size larger than $3.7 \mathrm{~cm}$ and heterogeneous Vimentin immunostaining.

p110 $\alpha$, PTEN and p-Akt are major signaling components in the pathway leading to mTOR activation, and were included in our study because of their importance in the molecular profiling of CCRCC and their emerging utility as prognostic markers and therapeutic targets [48-51]. p110 $\alpha$ is a major catalytic subunit of the PI3K enzyme and displays pro-oncogenic properties by virtue of generation of the phospho-inositide PIP3, which 
favors the phosphorylation and activation of the Akt effector kinase. Phospho-Akt (p-Akt) executes prooncogenic functions by phosphorylation of multiple protein substrates, being an important upstream activator of mTOR. This effect is counteracted by the action of the PTEN PIP3 phosphatase [52-54]. Most of the tumor samples analyzed in our study displayed p110 $\alpha$ positive and PTEN negative immunostaining, in agreement with their respective oncogenic and tumor suppressive functions in renal cancer [55]. However, we did not find correlation between PTEN and p-Akt (as a measurement of Akt activation) immunostaining in most of the tumors, suggesting the existence of PTEN-independent mechanisms driving Akt phosphorylation and activation in CCRCC, as previously proposed by others [56]. Most cases (33/40) displayed homogenous positive staining for p110 $\alpha$, whereas the majority of samples were homogenously negative for PTEN (26/40) or p-Akt (28/40). Classification analysis with these markers was not relevant for ITH.

SETD2 is the main methyltransferase responsible for trimethylation of histone- 3 at lysine-36 and is encoded in chromosome 3, whose $\mathrm{LOH}$ has been reported to be an early event in CCRCC [4]. In spite of the fact that this gene is mutated in 4-8\% of CCRCC [3], there is no evidence that these mutations carry clinical significance [57]. Very recent studies have shown that SETD2 loss of function causes dysfunctional DNA replication and repair, which promotes subclonal diversification. This contributes to ITH acting at early branches of the tumor phylogenetic tree [5]. We have detected a heterogeneous immunostaining of SETD2 protein in almost half of the cases (42.5\%), but SETD2 was an irrelevant variable for classification of IHT and grade.

\section{Conclusions}

ITH is a common event in CCRCC, even in organconfined tumors. Aside from the molecular approaches, conventional histologic and immunohistochemical studies may also demonstrate ITH in CCRCC, as revealed in this study. For such a purpose, Snail immunostaining appears to be an important marker. The high incidence of ITH in CCRCC recommends a wide sampling of the surgical specimens to guarantee that crucial data for the patient are not overlooked.

\footnotetext{
Abbreviations

CART: Classification and regression tree algorithm; CCRCC: Clear cell renal cell carcinoma; EMT: Epithelial to mesenchymal transition; HE: Hematoxylin-eosin staining; ITH: Intratumor heterogeneity; LOH: Loss of heterozygosity; MET: Mesenchymal to epithelial transition; TMA: Tissue microarray; WEKA: Waikato Environment for Knowledge Analysis.
}

\section{Competing interests}

The authors declare that they have no competing interests.

\section{Authors' contributions}

$R G, R P$ and JIL conceived the study, LZ obtained the clinical data from the clinical records and reviewed all hematoxylin-eosin sections, RG, JIL and LZ performed the immunohistochemical analysis, AE and JMC performed the statistical analysis, AE, JMC, RP and JIL contextualized statistical data with clinical settings, LZ wrote the draft, LZ, RG, RP and JIL wrote the final version; all authors read and approved the final manuscript.

\section{Acknowledgements}

The authors thank the excellent immunohistochemical work of Mar González, Alicia Esteve, Aida Larrañaga and Maria Cruz Andreu, Lab Technicians at the Department of Pathology, Cruces University Hospital, Barakaldo, Bizkaia, Spain. This work was partially funded by grant SAF201348812-R from Ministerio de Economía y Competitividad (Spain).

\section{Author details}

${ }^{1}$ Department of Pathology, Cruces University Hospital, University of the Basque Country (UPV/EHU), Plaza de Cruces s/n, 48903 Barakaldo, Bizkaia, Spain. ${ }^{2}$ Quantitative Biomedicine Unit, Biocruces Health Research Institute, Barakaldo, Bizkaia, Spain. ${ }^{3}$ Department of Pathology, Complejo Hospitalario de Navarra-Hospital Virgen del Camino, Pamplona, Navarra, Spain.

${ }^{4}$ Biomarkers in Cancer Unit, Biocruces Health Research Institute, Barakaldo, Bizkaia, Spain. ${ }^{5}$ kerbasque, Basque Foundation for Science, Bilbao, Bizkaia, Spain. ${ }^{6}$ Department of Cell Biology and Histology, University of the Basque Country (UPV/EHU), 48940 Leioa, Spain.

Received: 28 July 2015 Accepted: 1 March 2016

Published online: 08 March 2016

\section{References}

1. Siegel RL, Miller KD, Jemal A. Cancer statistics, 2015. CA Cancer J Clin. 2015; 65(1):5-29.

2. MacLennan GT, Cheng L. Neoplasms of the kidney. In: Bostwick DG, Cheng L, editors. Urologic Surgical Pathology. 3rd ed. Philadelphia: Elsevier Saunders; 2014. p. 76-156.

3. Gerlinger M, Rowan AJ, Horswell S, Larkin J, Endesfelder D, Gronroos E, et al. Intratumor heterogeneity and branched evolution revealed by multiregion sequencing. N Engl J Med. 2012;366(10):883-92.

4. Gerlinger M, Horswell S, Larkin J, Rowan AJ, Salm MP, Varela I, et al. Genomic architecture and evolution of clear cell renal cell carcinomas defined by multiregion sequencing. Nat Genet. 2014;46(3):225-33.

5. Kanu N, Grönross E, Martínez P, Burrell RA, Goh WY, Bartkova J, et al. SETD2 loss-of function promotes renal cancer branched evolution through replication stress and impaired DNA repair. Oncogene. 2015. doi:10.1038/ onc.onc2015.24.

6. De la Fuente IM. Metabolic dissipative structures. In: Aon MA, Saks V, Schlattner U, editors. Systems Biology of Metabolic and Signaling Networks: Energy, Mass and Information Transfer, Springer Series in Biophysics 16. New York: Springer; 2014. p. 179-212.

7. Gerlinger M, Catto JW, Orntoft TF, Real FX, Zwarthoff EC, Swanton C. Intratumor heterogeneity in urologic cancers: From molecular evidence to clinical implications. Eur Urol. 2015;67(4):729-37.

8. Audenet F, Yates DR, Cancel-Tassin G, Cussenot O, Rouprêt M. Genetic pathways involved in carcinogenesis of clear cell renal cell carcinoma: genomics towards personalized medicine. BJU Int. 2011;109(12):1864-70.

9. Sankin A, Hakimi AA, Mikkilineni N, Ostrovnaya I, Silk MT, Liang Y, et al. The impact of genetic heterogeneity on biomarker development in kidney cancer assessed by multiregional sampling. Cancer Med. 2014:3(6):1485-92.

10. López Jl, Guarch R, Camarasa N, Cáceres M, Moreno V, Muñiz G, et al. Grade heterogeneity in clear cell renal cell carcinoma. BJU Int. 2012. doi:10.1002/ BJUlw-2012-091-web.

11. López Jl, Guarch R, Larrinaga G, Corominas-Cishek A, Orozco R. Cell heterogeneity in clear cell renal cell carcinoma. APMIS. 2013;121(12):1187-91.

12. Zaldumbide L, Erramuzpe A, Guarch R, Cortés JM, López JI. Large $(>3.8 \mathrm{~cm})$ clear cell renal cell carcinomas are morphologically and immunohistochemically heterogeneous. Virchows Archiv. 2015;466(1):61-6.

13. Hall M, Frank E, Holmes G, Pfahringer B, Reutemann P, Witten IH. The WEKA Data Mining Software: An Update. SIGKDD Explor Newsl. 2009;11(1):10-8.

14. Fuhrman SA, Lasky LC, Limas C. Prognostic significance of morphologic parameters in renal cell carcinoma. Am J Surg Pathol. 1982;6(7):655-63. 
15. Gulati S, Martinez P, Joshi T, Birkbak NJ, Santos CR, Rowan AJ, et al. Systematic evaluation of the prognostic impact and intratumour heterogeneity of clear cell renal cell carcinoma biomarkers. Eur Urol. 2014;66(5):936-48.

16. Nassar A, Radhakrishnan A, Cabrero IA, Cotsonis GA, Cohen C. Intratumoral heterogeneity of immunohistochemical marker expression in breast carcinoma. A tissue microarray-based study. Appl Immunohistochem Mol Morphol. 2010;18(5):433-41.

17. Higgins JP, McKenney JK, Brooks JD, Argani P, Epstein JI. Recommendations for the reporting of surgically resected specimens of renal cell carcinoma: the Association of Directors of Anatomic and Surgical Pathology. Hum Pathol. 2009;40(4):456-63.

18. Algaba F, Delahunt B, Berney DM, Camparo P, Comperat E, Griffiths D, et al. Handling and reporting of nephrectomy specimens for adult renal tumours: a survey by the European Network of Uropathology. J Clin Pathol. 2012; 65(2):106-13.

19. Trpkov K, Grignon DJ, Bonsib SM, Amin MB, Billis A, Lopez-Beltran A, et al. Handling and staging of renal cell carcinoma: The International Society of Urological Pathology Consensus (ISUP) conference recommendations. Am J Surg Pathol. 2013;37(10):1505-17.

20. Kalluri R, Weinberg RA. The basics of epithelial-mesenchymal transition. J Clin Invest. 2009:119(6):1420-8.

21. Thiery JP, Acloque H, Huang RYJ, Nieto MA. Epithelial-mesenchymal transitions in development and disease. Cell. 2009;139(5):871-90.

22. Christiansen JJ, Rajasekaran AK. Reassessing epithelial to mesenchymal transition as a prerequisite for carcinoma invasion and metastasis. Cancer Res. 2006;66(17):8319-26.

23. Hugo H, Ackland ML, Blick T, Lawrence MG, Clements JA, Williams ED, et al. Epithelial-mesenchymal and mesenchymal-epithelial transitions in carcinoma progression. J Cell Physiol. 2007;213(2):374-83.

24. De Wever O, Pauwels P, De Craene B, Sabbah M, Emami S, Redeuilh G, et al. Molecular and pathological signatures of epithelial-mesenchymal transitions at the cancer invasion front. Histochem Cell Biol. 2008;130(3):481-94.

25. Klymkowsky MW, Savagner P. Epithelial-mesenchymal transition. A cancer researcher's conceptual friend and foe. Am J Pathol. 2009;174(5):1588-93.

26. Iwatsuki M, Mimori K, Yokobori T, Ishi H, Beppu T, Nakamori S, et al. Epithelial-mesenchymal transition in cancer development and its clinical significance. Cancer Sci. 2010;101(2):293-9.

27. He H, Magi-Galluzzi C. Epithelial-to-mesenchymal transitions in renal neoplasms. Adv Anat Pathol. 2014;21(3):174-80.

28. Zeisberg M, Neilson EG. Biomarkers for epithelial-mesenchymal transitions. J Clin Invest. 2009;119(6):1429-37.

29. Ohba K, Miyata Y, Matsuo T, Asai A, Mitsunari K, Shida Y, et al. High expression of Twist is associated with tumor aggressiveness and por prognosis in patients with renal cell carcinoma. Int J Clin Exp Pathol. 2014;7(6):3158-65

30. Harada Kl, Miyake H, Kusuda Y, Fujisawa M. Expression of epitelialmesenchymal transition markers in renal cell carcinoma: impact and prognostic outcomes in patients undergoing radical nephrectomy. BJU Int 2012:110(11PtC):E1131-37.

31. Fang Y, Wei J, Cao J, Zhao H, Liao B, Qiu S, et al. Protein expression of ZEB2 in renal cell carcinoma and its prognostic significance in patient survival. PLOS ONE. 2013;8(5):e62558.

32. Kaufhold S, Bonavida B. Central role of Snail1 in the regulation of EMT and resistance in cancer: a target for therapeutic intervention. J Exp Clin Cancer Res. 2014:33:62.

33. Lin TC, Liu YP, Chan YC, Su CY, Lin YF, Hsu SL, et al. Ghrelin promotes renal cell carcinoma metastasis via Snail activation and is associated with poor prognosis. J Pathol. 2015;237(1):50-61.

34. Mikami S, Katsube Kl, Oya M, Ishida M, Kosaka T, Mizuno R, et al. Expression of Snail and Slug in renal cell carcinoma: E-cadherin represso Snail is associated with cancer invasion and prognosis. Lab Invest. 2011; 91(10):1443-58

35. Liu W, Liu Y, Liu H, Zhang W, An H, Xu J. Snail predicts recurrence and survival of patients with localized clear cell renal cell carcinoma after surgical resection. Urol Oncol. 2015;33(2):69.

36. Zhu Y, Xu L, Zang J, Xu W, Liu Y, Yin H, et al. Klotho suppresses tumor progression via inhibiting PI3K/Akt/GSK3 $\beta / S n a i l$ signaling in renal cell carcinoma. Cancer Sci. 2013;104(6):663-71.

37. Thisse B, el Messal M, Perrin-Schmitt F. The twist gene: isolation of a Drosophila Zygotic gene necessary for the establishment of dorsoventral pattern. Nucleic Acids Res. 1987;15(8):3439-53.
38. Chen D, Gassenmaier M, Maruschke M, Riesenberg R, Pohla H, Stief CG, et al. Expression and prognostic significance of a comprehensive epithelia-mesenchymal transition gene set in renal cell carcinoma. J Urol. 2014;191(2):479-86.

39. Bullock MD, Sayan AE, Packham GK, Mirnezami AH. MicroRNAs: critical regulators of epithelial to mesenchymal (EMT) and mesenchymal to epithelial transition (MET) in cancer progression. Biol Cell. 2012;104(1):3-12.

40. Duns G, van den Berg A, van Dijk MC, van Duivenbode I, Giezen C, Kluiver J, et al. The entire miR-200 seed family is strongly deregulated in clear cell renal cell cancer compared to the proximal tubular epithelial cells of the kidney. Genes Chromosomes Cancer. 2013;52(2):165-73.

41. Lopez Jl. Renal tumors with clear cells. A review. Pathol Res Pract. 2013; 209(3):137-46.

42. Lawrie CH, Larrea E, Larrinaga G, Goicoechea I, Cáceres F, Arestin M, et al. Targeted next generation sequencing and non-coding RNA expression analysis of clear cell papillary renal cell carcinoma suggest distinct pathological mechanisms from other renal tumour types. J Pathol. 2014; 232(1):32-42.

43. Bienz M. beta-Catenin: a pivot between cell adhesion and Wnt signaling. Curr Biol. 2005:15(2):R64-7.

44. Brabletz T, Jung A, Hermann K, Günther K, Hohenberger W, Kirchner T. Nuclear overexpression of the oncoprotein beta-catenin in colorectal cancer is localized predominantly at the invasion front. Pathol Res Pract. 1998; 194(10):701-4.

45. Krabbe LM, Westerman ME, Bagrodia A, Gayed BA, Darwish OM, Haddad $A Q$, et al. Dysregulation of $\beta$-catenin is an independent predictor of oncologic outcomes in patients with clear cell renal cell carcinomas. J Urol. 2014;191(6):1671-7.

46. Lee JM, Dedhar S, Kalluri R, Thompson EW. The epithelial mesenchymal transition: new insights in signalling, development, and disease. J Cell Biol. 2006;172(7):973-81.

47. Truong LD, Shen SS. Immunohistochemical diagnosis of renal neoplasms. Arch Pathol Lab Med. 2011:135(1):92-109.

48. Sourbier C, Lindner V, Lang H, Agouni A, Schordan E, Danilin S, et al. The phosphoinositide 3-kinase/Akt pathway: a new target in human renal cell carcinoma therapy. Cancer Res. 2006;66(10):5130-42.

49. Mersenburger AS, Hennenlotter J, Kuehs U, Simon P, Kruck S, Koch E, et al. Activation of PI3K is associated with reduced survival in renal cell carcinoma. Urol Int. 2008;80(4):372-7.

50. Darwish OM, Kapur P, Youssef RF, Bagrodia A, Belsante M, Alhalabi F, et al. Cumulative number of altered biomarkers in mammalian target of rapamycin pathway is an idependent predictor of outcome in patients with clear cell renal cell carcinoma. Urology. 2013;81(3):581-6.

51. Porta C, Figlin RA. Phosphatidylinositol-3-kinase/Akt signaling pathway and kidney cancer, and the therapeutic potential of phosphatidylinositol-3kinase/Akt inhibitors. J Urol. 2009;182(6):2569-77.

52. Carnero A, Blanco-Aparicio C, Renner O, Link W, Leal JF. The PTEN/PI3K/AKT signaling pathway in cancer, therapeutic implications. Curr Cancer Drug Targets. 2008;8(3):187-98

53. Yuan TL, Cantley LC. PI3K pathway alterations in cancer: variations on a theme. Oncogene. 2008;27(41):5497-510.

54. Pulido R. PTEN: a yin-yang master regulator protein in health and disease. Methods. 2015;77-78:3-10.

55. Shin Lee J, Seok Kim H, Bok Kim Y, Cheol Lee M, Soo PC. Expression of PTEN in renal cell carcinoma and its relation to tumor behavior and growth. J Surg Oncol. 2003;84(3):166-72.

56. He L, Fan C, Gillis A, Feng X, Sanatani M, Hotte S, et al. Co-existence of high levels of the PTEN protein with enhanced Akt activation in renal cell carcinoma. Biochim Biophys Acta. 2007:1772(10):1134-42.

57. Gossage L, Murtaza M, Slatter AF, Lichtenstein CP, Warren A, Haynes B, et al. Clinical and pathological impact of VHL, PBRM1, BAP1, SETD2, KDM6A, and JARID1C in clear cell renal cell carcinoma. Genes Chromosomes Cancer. 2014:53(1):38-51. 